\title{
DETERMINAÇÃO DA DENSIDADE BÁSICA DA MADEIRAS DE EUCALYPTUS POR DIFERENTES MÉTODOS NÃO DESTRUTIVOS ${ }^{1}$
}

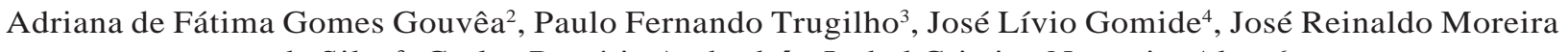
da Silva ${ }^{3}$, Carlos Rogério Andrade ${ }^{5}$ e Isabel Cristina Nogueira Alves ${ }^{6}$

\begin{abstract}
RESUMO - Os métodos não destrutivos vêm se tornando importante alternativa para predições das características da madeira. A facilidade e precisão das predições têm feito com que muitas empresas adotem estes novos métodos. A avaliação da qualidade da madeira por técnicas mais simples e rápidas é uma necessidade fundamental na qualificação de florestas de Eucalyptus. O objetivo deste trabalho foi investigar diferentes métodos de avaliação não destrutiva da madeira, a saber: (1) Resistógrafo, (2) Pilodyn e (3) Espectroscopia de Infravermelho Próximo-NIRS para predição da densidade básica da madeira. Neste estudo foram utilizados seis clones de eucalipto, com 3 anos de idade, plantados comercialmente com espaçamentos de 3,0 x 3,3m. As amostras foram coletadas no Estado de Minas Gerais, em diferentes regiões (Cocais, Guanhães, Rio Doce e Santa Bárbara). Foram utilizadas cinco árvores por clone. A técnica para estimativa da densidade básica que apresentou maior precisão foi a resistografia, seguida do NIR e, finalmente, do Pilodyn.
\end{abstract}

Palavras-chave: Resistógrafo, Eucalyptus, Ensaio Não Destrutivo, NIRS, Densidade Básica e Pilodyn.

\section{DETERMINATION OF EUCALYPTUS BASIC DENSITY BY DIFFERENT NON- DESTRUCTIVE METHODS}

\begin{abstract}
Non-destructive methods are becoming an important alternative for predicting the wood characteristics. The predictions ease and accuracy have led many companies to adopt these new methods. Assessing the wood quality by simpler and faster techniques is a fundamental need in the Eucalyptus forests qualification. This study aimed to investigate some different methods of wood nondestructive evaluation, namely: "(1) Resistograph, (2) Pilodyn, and (3), and Near Infrared Spectroscopy (NIRS)" to predict the wood basic density. Six different three-year-old eucalyptus clones, planted commercially in the spacing of $3.0 \times 3.3$, were used for this study. The samples were collected in Minas Gerais, from different regions (Cocais, Guanhães, Rio Doce, and Santa Bárbara). We used five trees per clone. The technique to estimate the basic density with the highest accuracy was resistograph followed by the NIR and finally Pilodyn.
\end{abstract}

Keywords: Resistograph, Eucalyptus, Nondestructive testing, NIRS. Basic density and Pilodyn

\footnotetext{
${ }^{1}$ Recebido em 18.12.2008 e aceito para publicação em 16.12.2010.

${ }^{2}$ Programa de Pós-Graduação em Ciência Florestal na Universidade Federal de Viçosa, UFV, Brasil. E-mail: $<$ agomes@ufv.br> .

${ }^{3}$ Universidade Federal de Lavras, UFLA, Brasil. E-mail:<trugilho@dcf.ufla.br>e <jreinaldo@dcf.ufla.br>.

${ }^{4}$ Universidade Federal de Viçosa, UFV, Brasil. E-mail: <jlgomide@ufv.br>.

${ }^{5}$ Universidade Federal de Goiás, UFG, Brasil. E-mail:<cra.florestal@yahoo.com.br>.

${ }^{6}$ Programa de Pós-Graduação em Tecnologia da Madeira pela Universidade Federal de Lavras, UFLA, Brasil. E-mail:

<bebelcrist@yahoo.com.br>.
} 


\section{INTRODUÇÃO}

A qualidade da madeira de Eucalyptus tem sido amplamente estudada no Brasil nas últimas décadas, e sua importância e impactos na produção industrial de celulose foram demonstrados por vários estudos, como os realizados por Gomide (2004 e 2005).

A avaliação não destrutiva da madeira para determinação de suas características tecnológicas é importante ferramenta para o conhecimento da variabilidade entre indivíduos e a caracterização de material no campo. As técnicas não destrutivas têm sido cada vez mais utilizadas por vários setores florestais e industriais.

Várias pesquisas têm sido desenvolvidas com o objetivo de predizer propriedades da madeira por meio de métodos não destrutivos. Trabalhos de Souza et al. (2003), Cardoso Júnior et al. (2005), Pádua et al. (2004) e Trugilho et al. (2006) são alguns exemplos da utilização de métodos não destrutivos na avaliação da madeira.

Para Hein et al. (2009), a técnica da espectroscopia no infravermelho próximo é técnica de análise eficiente e que pode ser usada para estimativa da densidade básica da madeira de E. Urophylla e E. grandis. Esse autor encontrou coeficientes de determinação dos modelos de predição variando entre 0,74 e 0,86 e a relação de desempenho do desvio (RPD) entre 1,9 e 2,7. Santos et al. (2009), avaliando a densidade básica da madeira de 30 amostras de Eucalyptus, em forma de cavacos, obtiveram correlações elevadas (97\%) e baixos erros médios de predição (RMSEP), de apenas 7,7 e $7,8 \mathrm{~kg} / \mathrm{m}^{3}$ para os teores de umidade de $33 \%$ e $5 \%$, respectivamente. Os resultados demonstram alta precisão obtida na predição da densidade básica da madeira, utilizando a espectroscopia no infravermelho próximo.

Essas pesquisas têm contribuído para o avanço do conhecimento da variabilidade natural da madeira, permitindo a identificação de material que possui madeira mais homogênea e livre de defeitos internos, o que auxilia na sua utilização mais adequada. Os ensaios não destrutivos apresentam como principais vantagens a rapidez na coleta de dados, o baixo custo e a possibilidade de avaliação de grande número de amostras, o que favorece a seleção de genótipos superiores, mesmo em condição de campo.
O objetivo deste estudo foi caracterizar a madeira de clones de Eucalyptus por meio de métodos de avaliações não destrutivas, incluindo o uso de resistógrafo, pilodyn e espectroscopia no infravermelho próximo.

\section{MATERIAL E MÉTODOS}

Foram utilizados neste estudo seis clones de Eucalyptus com 3 anos de idade, plantados em parcelas de $10 \times 10$ plantas, com espaçamento de 3,0 x 3,3 m, provenientes de quatro diferentes locais do Estado de Minas Gerais (Cocais, Guanhães, Rio Doce e Santa Bárbara). Os seguintes clones foram analisados: clone 0057 (híbrido natural de E.grandis), clone 1046 (híbrido de E.grandis x E. urophylla), clone 1213 (híbrido de E.grandis x E. urophylla), clone 1215 (híbrido de E.grandis x E. urophylla), clone 1274 (híbrido de E.grandis x E. urophylla) e clone 7074 (Eucalyptus grandis). Selecionaram-se cinco árvores de cada clone, que apresentavam diâmetro médio \pm desvio-padrão. Foram coletadas cinco árvores, por clone e local, num total de 120 amostras.

\section{Determinação convencional da densidade básica da madeira}

Foram determinadas as densidades básicas das madeiras (Db) utilizando dois diferentes métodos de amostragem: amostra em forma de disco de madeira coletado a 1,3 m de altura do solo (DbDAP) e amostra de cavacos produzidos com a árvore (DbM). A densidade no DAP foi determinada em um disco de $2,5 \mathrm{~cm}$ de espessura, utilizando-se o método da medição indireta do volume (balança hidrostática), descrito pela Norma TAPPI 258 om-85. A densidade básica dos cavacos, denominada densidade básica média da árvore (DbM), foi determinada conforme Norma da ABNT, utilizando cavacos produzidos com cinco toretes de $100 \mathrm{~cm}$ retirados na base, a 25\%, $50 \%, 75 \%$ e $100 \%$ da altura comercial do tronco, considerada até um diâmetro mínimo de $7 \mathrm{~cm}$. A densidade básica determinada no disco de madeira foi obtida pela média de duas cunhas opostas e a dos cavacos, de amostra composta, representada pela mistura de todos os pontos da amostragem longitudinal no tronco das árvores.

\section{Determinação da densidade básica pelo resistógrafo}

O Resistographâ (resistógrafo) mede a resistência da madeira à penetração de uma broca especial de 3 mm de diâmetro e comprimento variável. Essa resistência pode ser correlacionada com a densidade básica da madeira, e, assim, medições rápidas com o resistógrafo podem predizer a sua densidade básica. 
As medições foram realizadas na altura do DAP, nas posições entre e dentro das linhas de plantio, no mesmo local onde foi realizada posteriormente a retirada do disco para determinação da densidade básica na altura do DAP (DbDAP). As leituras foram realizadas após a retirada da casca, por toda a sua circunferência, e posteriormente as árvores foram derrubadas para produção de cavacos. O resistógrafo permite estimar a densidade básica, a sanidade (ataque de brocas e cupins) e as propriedades mecânicas da madeira.

\section{Determinação da densidade básica pelo Pilodyn ${ }^{\circledR}$}

As medições com o pilodyn, que mede a resistência à penetração na madeira do pino desse aparelho, foram realizadas nas mesmas posições das feitas com o resistógrafo. As medidas foram tomadas na circunferência do tronco das árvores vivas, ou seja, duas opostas dentro da linha e duas opostas entre as linhas de plantio. O procedimento adotado foi o descrito por Oliveira (2001).

\section{Determinação da densidade básica por espectroscopia no infravermelho próximo-NIRS}

Amostras de serragem foram retiradas na altura do DAP, com furadeira manual dotada de broca. As serragens foram processadas em moinho tipo ciclone para obtenção de partículas com granulometria de aproximadamente $0,05 \mathrm{~mm}$. Acondicionaram-se as amostras em sala climatizada $\left(25^{\circ} \mathrm{C}\right.$ e $40 \%$ de umidade relativa), antes da obtenção dos espectros infravermelhos, utilizando espectrômetro marca FOSS, modelo NirSystem 5000, que opera na região espectral de 1.100 a $2.500 \mathrm{~nm}$. Os espectros foram coletados em intervalos de $2 \mathrm{~nm}$, totalizando 700 valores de absorbância. Os espectros utilizados para estabelecimento dos modelos de calibração do espectrofotômetro foram provenientes da média de duas leituras. A partir dos espectros obtidos das 120 amostras, foram selecionados 96 espectros para a geração de um modelo matemático para predição da densidade básica, e 24 espectros foram utilizados para validação externa.

\section{Calibração e geração de modelos NIRS de predição da densidade básica}

$\mathrm{Na}$ construção e validação dos modelos quimiométricos para predição das densidades básicas das madeiras foi utilizado o software Unscrambler, versão 9.6, e a metodologia seguiu o esquema apresentado na Figura 1.

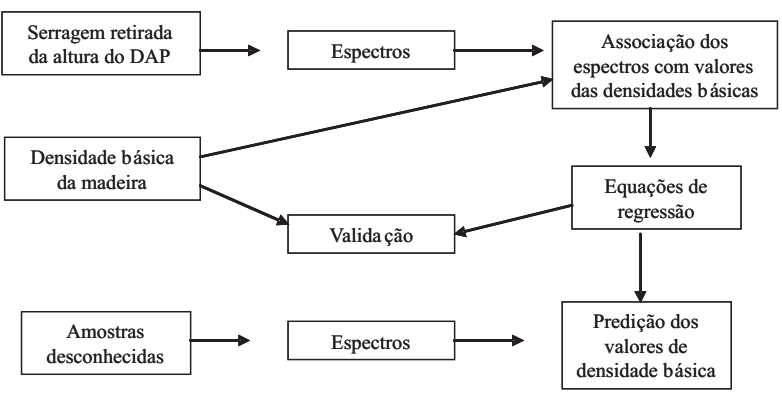

Figura 1 - Esquema de calibração, validação e predição pela técnica NIRS.

Figure 1 - Calibration scheme, validation and prediction by NIRS technique.

O pré-tratamento matemático utilizado foi a primeira derivada para a retirada de ruídos. Depois da retirada das amostras consideradas “outliers”, por meio da análise de componentes principais (PCA) e escores, foi empregada a técnica de regressão pelo método dos mínimos quadrados parciais (PLS), que seleciona coeficientes que minimizam a soma dos quadrados dos resíduos (na matriz de $\mathrm{x}=700$ ). A análise de componentes principais (PCA) é técnica estatística utilizada para reduzir o número de variáveis e fornecer visão estatisticamente privilegiada do conjunto de dados. A análise de componentes principais fornece informações necessárias para identificar as variáveis mais importantes no espaço das componentes principais. As variáveis que são os chamados de fatores correspondem ao número de componentes principais necessários para descrever o conjunto de dados na regressão PLS (mínimos quadrados parciais). A escolha não adequada do número de fatores pode resultar em desajuste no modelo.

A mensuração de como a validação se ajusta aos dados é representada pelo $\mathrm{R}^{2}$ e RMSEP. O R $\mathrm{R}^{2}$ indica o grau de associação entre os valores obtidos nas análises tradicionais de laboratório e os valores preditos nos modelos gerados. Para obter uma curva de calibração com alta precisão, esse valor deve ser mais próximo de 1. O RMSEP (equação 1) é o erro da predição, proveniente da validação cruzada, que consiste na retirada de uma amostra por vez, gerando modelo com o restante e predizendo a amostra retirada, ou da validação externa, que consiste na predição de um novo conjunto de amostras e na comparação desses resultados com os valores de laboratório. O RMSEC (equação 2) é o erro da calibração calculado com amostras presentes no modelo, constituindo a média dos desvios dos 
resultados de laboratório e NIRS. Os valores RMSEP e RMSEC devem ser o mais próximo de zero.

(equação 1)

RMSEP = erro-padrão da predição

$S E P=\sqrt{\frac{\sum_{i=1}^{N P}\left(\hat{y}_{i}-y_{i}\right)^{2}}{(N P-1)}}$

em que:

$\hat{y}=$ valor do constituinte de interesse da amostra i, predita pela calibração;

$y_{i}=$ valor de referência da amostra i, ou seja, o valor conhecido; e

NP = número de amostras da predição.

(equação 2)

RMSEC = erro-padrão da calibração

$S E C=\sqrt{\frac{\sum_{i=1}^{N C}\left(\hat{y}_{i}-y_{i}\right)^{2}}{(N C-K-1)}}$

em que:

$\hat{y}=$ valor do constituinte de interesse para a validação da amostra i, estimado usando a calibração;

$y_{i}=$ valor conhecido do constituinte de interesse da amostra i;

$\mathrm{NC}=$ número de amostras usadas para o desenvolvimento da calibração; e

$\mathrm{K}=$ número de fatores usados para o desenvolvimento da calibração.

\section{Análise estatística}

Na avaliação estatística, utilizou-se o delineamento experimental inteiramente casualizado disposto em esquema fatorial com dois fatores (clones e locais) e cinco repetições (árvores-amostra).

\section{RESULTADOS E DISCUSSÃO}

\section{Densidade básica da madeira}

As médias das densidades básicas dos clones e o teste de comparação múltipla encontram-se na Tabela 1.

As médias das densidades básicas dos discos de madeira no DAP (DbDAP) e as densidades básicas médias das árvores (DbM), determinadas nos cavacos, foram bastante semelhantes em todos os clones, com diferenças de apenas 0,004-0,029 g/ $\mathrm{cm}^{3}$, ou seja, $1 \mathrm{a}$ $6 \%$. Os efeitos de clone e de local foram não significativos para DbDAP, mas significativos para a DbM, a 1\% de probabilidade. Interação significativa indica a existência de dependência entre os fatores, e, por isso, optou-se por fazer o desdobramento dessa interação significativa e a avaliação do efeito do local no clone, como mostrado na Tabela 2.

Na Figura 2 são mostradas as duas técnicas de amostragem para determinação de densidade básica das árvores, o disco no DAP e os cavacos amostrando a árvore integral. Essas duas técnicas apresentaram baixa correlação (30\%), mostrando que a densidade básica na altura do DAP não prediz, com precisão aceitável, a densidade média da árvore. Entretanto, estudo realizado com 545 árvores, com idades variando de 6 a 9 anos, coletadas em diferentes regiões do Estado de Minas Gerais, demonstrou alto coeficiente de determinação (84\%), entre a densidade básica medida no DAP e a densidade básica média ponderada da árvore inteira determinada em cinco discos obtidos em diferentes alturas do tronco (MILAGRES, 2008). O número relativamente baixo de amostras e a alta variabilidade verificada neste estudo poderiam ser uma explicação para a baixa correlação encontrada, e, portanto, novos estudos são recomendados para esclarecimento dessa divergência de resultados.

\section{Resistógrafo e Pilodyn}

Os valores médios das leituras realizadas com o resistógrafo (Res) e o pilodyn (Pil), por clone e local de amostragem, além do teste de comparação múltipla realizado, encontram-se na Tabela 3. Numa rápida análise comparativa, pode ser observado que, de maneira geral, existe correlação entre a densidade básica da madeira (Tabela 1) e as resistências à penetração da broca ou pinos desses aparelhos (Tabela 3). Nesta tabela pode ser verificado que o clone de menor densidade (7074) foi o que apresentou os maiores valores de penetração do pino do Pilodyn e os menores valores de amplitude.

Na Tabela 4, encontra-se o resumo da análise de variância do delineamento experimental adotado. Os efeitos de clone e local foram significativos para todas as características avaliadas, a 1\% de probabilidade, exceto para densidade básica no DAP, em que a relação foi não significativa. Interação significativa indica a 
Tabela 1 - Resultados médios e testes de comparação de médias da densidade básica dos cavacos e no DAP, por clone e local de amostragem, para os 24 clones/locais avaliados.

Table 1 - Mean results and comparison tests of mean chips wood basic density and in the diameter at breast height (DBH) per clone and sampling site of the 24 clones/assessed sites.

\begin{tabular}{|c|c|c|c|}
\hline Clones & Locais & $\operatorname{DbM}\left(\mathrm{g} / \mathrm{cm}^{3}\right)$ & $\operatorname{DbDAP}\left(\mathrm{g} / \mathrm{cm}^{3}\right)$ \\
\hline \multirow{5}{*}{57} & Cocais & 0,418 & 0,461 \\
\hline & Guanhães & 0,441 & 0,455 \\
\hline & Rio Doce & 0,444 & 0,455 \\
\hline & Santa Bárbara & 0,431 & 0,437 \\
\hline & Média & 0,434 & $0,452 \mathrm{~b}$ \\
\hline \multirow{5}{*}{1046} & Cocais & 0,434 & 0,472 \\
\hline & Guanhães & 0,439 & 0,449 \\
\hline & Rio Doce & 0,429 & 0,440 \\
\hline & Santa Bárbara & 0,437 & 0,429 \\
\hline & Média & 0,435 с & $0,448 \mathrm{~b}$ \\
\hline \multirow{5}{*}{1213} & Cocais & 0,453 & 0,511 \\
\hline & Guanhães & 0,467 & 0,460 \\
\hline & Rio Doce & 0,471 & 0,464 \\
\hline & Santa Bárbara & 0,456 & 0,446 \\
\hline & Média & 0,462 a & 0,470 a \\
\hline \multirow{5}{*}{1215} & Cocais & 0,430 & 0,422 \\
\hline & Guanhães & 0,459 & 0,450 \\
\hline & Rio Doce & 0,447 & 0,439 \\
\hline & Santa Bárbara & 0,466 & 0,438 \\
\hline & Média & $0,466 \mathrm{~b}$ & $0,437 \mathrm{~b}$ \\
\hline \multirow{5}{*}{1274} & Cocais & 0,439 & 0,545 \\
\hline & Guanhães & 0,464 & 0,461 \\
\hline & Rio Doce & 0,451 & 0,459 \\
\hline & Santa Bárbara & 0,431 & 0,427 \\
\hline & Média & $0,446 \mathrm{~b}$ & 0,437 a \\
\hline \multirow{5}{*}{7074} & Cocais & 0,372 & 0,379 \\
\hline & Guanhães & 0,372 & 0,375 \\
\hline & Rio Doce & 0,392 & 0,396 \\
\hline & Santa Bárbara & 0,355 & 0,357 \\
\hline & Média & $0,373 \mathrm{~d}$ & 0,377 с \\
\hline
\end{tabular}

DbM= densidade básica média dos cavacos; DbDAP= densidade básica no DAP.

Médias seguidas de mesma letra, dentro de clone, não diferem entre si, pelo teste de Scott-Knott, a 5\% de probabilidade.

existência de dependência entre os fatores. Dessa forma, optou-se por fazer o desdobramento da interação e avaliação do efeito do local (regional) dentro do clone.

Uma vez que as leituras de ambos os aparelhos, o resistógrafo e o pilodyn, demonstraram ter alguma correlação com a densidade básica da madeira, optou-se pelo desenvolvimento de modelos matemáticos para determinação da densidade básica da madeira por meio da utilização desses aparelhos. Nas Figuras 3 e 4, as correlações e respectivos modelos matemáticos são apresentados para as duas técnicas de determinação da densidade básica da madeira (DbM e DbDAP), bem como as leituras do resistógrafo (Res) e do pilodyn (Pil). O modelo estabelecido para o resistógrafo apresentou

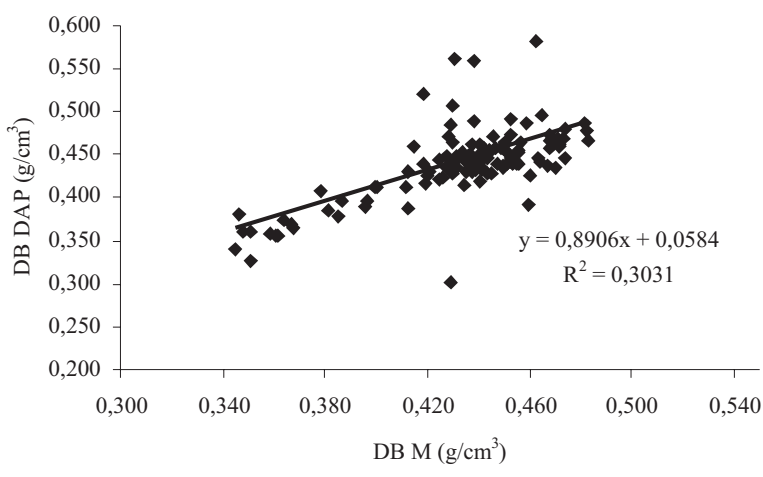

Figura 2 - Relação entre a densidade básica no DAP (DbDAP) e a densidade básica média da árvore (DbM).

Figure 2-Relationship between basic density in $\mathrm{DBH}(\mathrm{BdDBH})$ and the tree mean basic density (MBD).

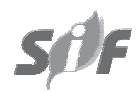

Revista Árvore, Viçosa-MG, v.35, n.2, p.349-358, 2011 
Tabela 2 - Resumo da análise de variância e do desdobramento da interação e avaliação do efeito de local dentro de clone para a densidade básica média (DbM) e densidade no DAP (DbDAP).

Table 2 - Analysis of variance and of the interaction unfolding; evaluation of the site effect within clone for the mean basic density $(M B D)$ and $D B H$ density $(B d D B H)$.

\begin{tabular}{|c|c|c|c|}
\hline \multirow[t]{2}{*}{ FV } & \multirow[t]{2}{*}{ GL } & \multicolumn{2}{|c|}{ Quadrado médio } \\
\hline & & DbM & DbDAP \\
\hline Clone(C) & 5 & $0,0191 * *$ & $0,0243 * *$ \\
\hline Local(L) & 3 & $0,0020 * *$ & $0,0106 * *$ \\
\hline C x L & 15 & $0,0003 * *$ & $0,0019 \mathrm{~ns}$ \\
\hline Resíduo & 96 & 0,0001 & 0,0014 \\
\hline Total & 119 & & \\
\hline Média & & 0,433 & 0,444 \\
\hline CV(\%) & & 2,71 & 8,43 \\
\hline \multirow[t]{2}{*}{ FV } & GL & \multicolumn{2}{|c|}{ Quadrado médio } \\
\hline & & \multicolumn{2}{|c|}{$\mathrm{DbM}$} \\
\hline Local/Clone 57 & 3 & \multicolumn{2}{|c|}{$0,0007 * *$} \\
\hline Local/Clone 1046 & 3 & \multicolumn{2}{|c|}{$0,0001 \mathrm{~ns}$} \\
\hline Local/Clone 1213 & 3 & \multicolumn{2}{|c|}{$0,0003 * *$} \\
\hline Local/Clone 1215 & 3 & \multicolumn{2}{|c|}{$0,0007 * *$} \\
\hline Local/Clone 1274 & 3 & \multicolumn{2}{|c|}{$0,0010 * *$} \\
\hline Local/Clone 7074 & 3 & \multicolumn{2}{|c|}{$0,0011 * *$} \\
\hline Resíduo & 96 & \multicolumn{2}{|c|}{0,0001} \\
\hline
\end{tabular}

DbM= densidade básica dos cavacos da árvore, DbDAP= densidade básica do disco no DAP, FV = fonte de variação, GL = grau de liberdade; **, * e ns = significativo a $1 \%$ e $5 \%$ de probabilidade e não significativo, respectivamente.

melhor coeficiente de determinação (74\%), enquanto no pilodyn foi de apenas $57 \%$. Esses valores indicam que o resistógrafo é mais adequado para estimar a densidade básica da madeira. A broca perfurante do resistógrafo perfura todo o diâmetro do tronco da árvore, enquanto as hastes do pilodyn penetram apenas pequena profundidade no tronco, o que explica a maior precisão do resistógrafo. Thiersch et al. (2006) encontraram equações de densidade precisas e exatas quando avaliaram o uso do pilodyn e de variáveis dendrométricas no desenvolvimento de modelos para estimar a densidade básica da madeira de clones de Eucalyptus spp.

As relações das densidades básicas da madeira com o pilodyn e o resistógrafo encontram-se ilustradas nas Figuras 3 e 4 . Os valores determinados com o resistógrafo encontram-se mais próximos da curva estimada que os do pilodyn. Entretanto, Greaves et al. (1996), em estudo com diferentes espécies de eucalipto, encontraram altas relações na densidade básica com os valores da penetração pelo pino do pilodyn.

Os modelos ajustados para determinação da densidade básica da madeira por meio dos valores obtidos com o resistógrafo e o pilodyn encontram-se na Tabela 5. Observa-se, nessa tabela, que o modelo usando o resistógrafo apresentou melhor ajuste e menor coeficiente de variação, indicando que esse equipamento é mais adequado para estimar o valor da densidade básica da madeira.

\section{Espectroscopia de infravermelho próximo (NIRS)}

Os dados espectrais para análise no NIRS foram tratados por análise multivariada, que corresponde a um grande número de métodos e técnicas, as quais utilizam simultaneamente todas as variáveis na interpretação teórica do conjunto de dados obtidos. Os espectros NIR das 120 amostras de serragem podem ser visualizados na Figura 5.

Na Tabela 5 são apresentadas as características do modelo matemático desenvolvido para predição da densidade básica da madeira pela técnica NIRS. Para o desenvolvimento desse modelo, do total de 120 amostras, 96 foram utilizadas para geração do modelo e 24 para validação externa.

Para analisar a precisão desse modelo foram realizadas validações externas com 24 amostras. A validação externa compara o resultado obtido pelas análises usuais de laboratório com o conseguido pelo modelo ajustado, usando amostras que não fizeram 
Tabela 3 - Resultados médios e testes de comparação de médias, das medições do resistógrafo (Res) e do pilodyn (Pil), por clone e local de amostragem, para os 24 clones/locais avaliados.

Table 3 - Mean results and comparison tests from the resistograph measurements (Res) and Pilodyn (Pil) per clone and sampling site of the 24 clones/assessed sites.

\begin{tabular}{|c|c|c|c|}
\hline Clones & Locais & Res (\%) & Pil (mm) \\
\hline \multirow{5}{*}{57} & Cocais & $14,6 \mathrm{~b}$ & 17,8 a \\
\hline & Guanhães & 17,0 а & $16,3 \mathrm{~b}$ \\
\hline & Rio Doce & 17,6 а & $13,8 \mathrm{c}$ \\
\hline & Sta Bárbara & 19,0 а & $16,4 \mathrm{~b}$ \\
\hline & Média & 17,1 & 16,1 \\
\hline \multirow{5}{*}{1046} & Cocais & 19,1 a & $16,2 \mathrm{~b}$ \\
\hline & Guanhães & 19,2 а & $16,3 \mathrm{~b}$ \\
\hline & Rio Doce & $13,0 \mathrm{~b}$ & $15,4 \mathrm{~b}$ \\
\hline & Sta Bárbara & 19,3 а & 17,4 a \\
\hline & Média & 17,7 & 16,3 \\
\hline \multirow{5}{*}{1213} & Cocais & $18,9 \mathrm{~b}$ & 18,5 a \\
\hline & Guanhães & $18,0 \mathrm{~b}$ & $16,8 \mathrm{~b}$ \\
\hline & Rio Doce & 22,6 a & $16,2 \mathrm{~b}$ \\
\hline & Sta Bárbara & 20,7 a & $17,1 \mathrm{~b}$ \\
\hline & Média & 20,1 & 17,2 \\
\hline \multirow{5}{*}{1215} & Cocais & 19,7 a & 18,1 a \\
\hline & Guanhães & 20,5 a & $16,4 \mathrm{~b}$ \\
\hline & Rio Doce & 20,4 a & $17,2 \mathrm{~b}$ \\
\hline & Sta Bárbara & 22,8 a & $16,7 \mathrm{~b}$ \\
\hline & Média & 20,9 & 17,1 \\
\hline \multirow{5}{*}{1274} & Cocais & $14,3 \mathrm{c}$ & 16,8 a \\
\hline & Guanhães & 23,7 a & $15,3 \mathrm{~b}$ \\
\hline & Rio Doce & $18,1 \mathrm{~b}$ & $15,3 \mathrm{~b}$ \\
\hline & Sta Bárbara & $19,5 \mathrm{~b}$ & 16,9 а \\
\hline & Média & 18,9 & 16,1 \\
\hline \multirow{5}{*}{7074} & Cocais & 9,8 a & 18,6 a \\
\hline & Guanhães & 10,6 a & 19,4 а \\
\hline & Rio Doce & 11,9 а & 19,2 а \\
\hline & Sta Bárbara & 8,0 a & 20,2 a \\
\hline & Média & 10,1 & 19,4 \\
\hline
\end{tabular}

Médias seguidas de mesma letra, dentro de clone, não diferem entre si, pelo teste de Scott-Knott, a 5\% de probabilidade.
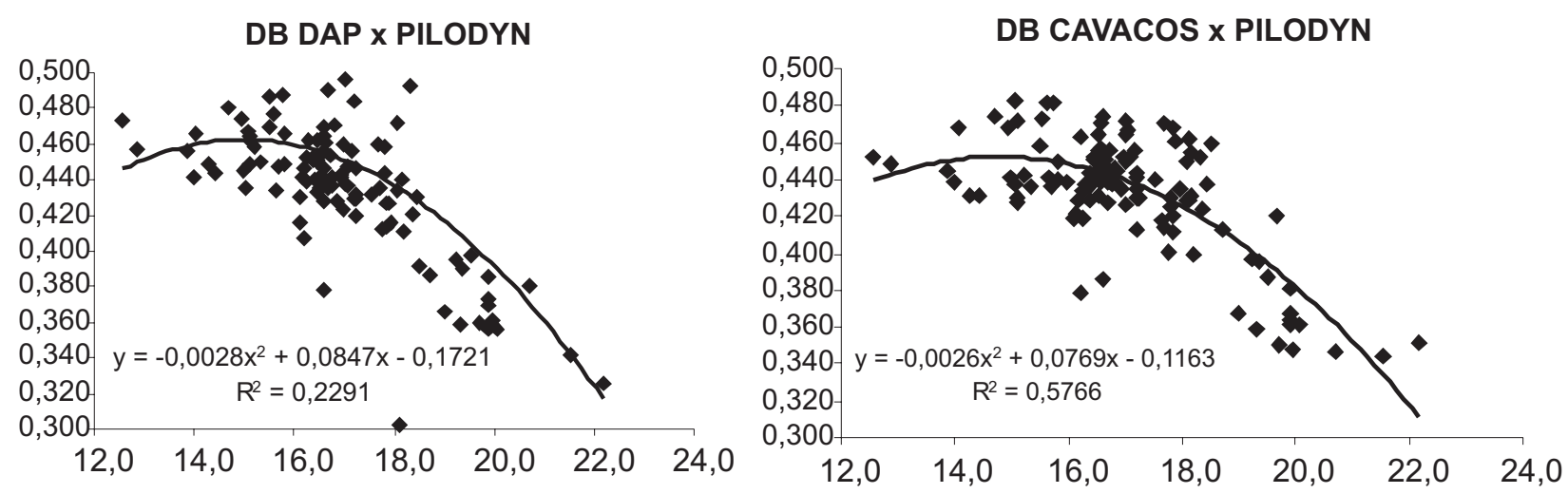

Figura 3 - Relação do pilodyn com as densidades básicas da madeira (Dbm e DbDAP).

Figure 3 - Relationship between Pilodyn with the wood basic density (Wbd and BdDBH). 
Tabela 4 - Resumo da análise de variância e do desdobramento da interação e avaliação do efeito de local dentro de clone para a densidade básica média (DbM), densidade no DAP (DbDAP), resistógrafo (Res) e resistência à penetração do pino do pilodyn (Pil).

Table 4 - Analysis of variance and of the interaction unfolding and evaluation of the site effect within clone for mean basic density (MBD), density in $D B H(B d D B H)$, resistograph (Res) and resistance to penetration by the Pilodyn (Pil) pin.

\begin{tabular}{|c|c|c|c|c|c|}
\hline \multirow[t]{2}{*}{ FV } & \multirow[t]{2}{*}{ GL } & \multicolumn{4}{|c|}{ Quadrado médio } \\
\hline & & $\mathrm{Db}_{\mathrm{M}}$ & $\mathrm{Db}_{\mathrm{DAP}}$ & RES & PIL \\
\hline Clone(C) & 5 & $0,0191 * *$ & $0,0243 * *$ & $299,0814 * *$ & $30,5947 * *$ \\
\hline Local(L) & 3 & $0,0020 * *$ & $0,0106 * *$ & $30,7180 * *$ & $13,7898 * *$ \\
\hline $\mathrm{C} \times \mathrm{L}$ & 15 & $0,0003 * *$ & $0,0019 \mathrm{~ns}$ & $30,8049 * *$ & $3,4728 * *$ \\
\hline Resíduo & 96 & 0,0001 & 0,0014 & 55,268 & 0,8077 \\
\hline Total & 119 & & & & \\
\hline Média & & 0,433 & 0,444 & 17,43 & 17,02 \\
\hline CV(\%) & & 2,71 & 8,43 & 13,49 & 5,28 \\
\hline \multirow[t]{2}{*}{$\mathrm{FV}$} & GL & & & Quadrado médi & \\
\hline & & \multicolumn{2}{|c|}{ DBM } & AMP & PIL \\
\hline Local/Clone 57 & 3 & \multicolumn{2}{|c|}{$0,0007 * *$} & $17,1233 *$ & $13,8246 * *$ \\
\hline Local/Clone 1046 & 3 & \multicolumn{2}{|c|}{$0,0001 \mathrm{~ns}$} & $48,3978 * *$ & $3,3260 * *$ \\
\hline Local/Clone 1213 & 3 & \multicolumn{2}{|c|}{$0,0003 * *$} & $21,19650 *$ & $4,8591 * *$ \\
\hline Local/Clone 1215 & 3 & \multicolumn{2}{|c|}{$0,0007 * *$} & 9,3893 ns & $2,9845 *$ \\
\hline Local/Clone 1274 & 3 & \multicolumn{2}{|c|}{$0,0010 * *$} & $75,2685 * *$ & $3,9045 * *$ \\
\hline Local/Clone 7074 & 3 & \multicolumn{2}{|c|}{$0,0011 * *$} & $13,3673 \mathrm{~ns}$ & $2,2551 *$ \\
\hline Resíduo & 96 & \multicolumn{2}{|c|}{0,0001} & $5,526,875$ & 0,8077 \\
\hline
\end{tabular}

Res = amplitude do resistógrafo, Pi l= penetração do pino do pilodyn, FV = fonte de variação, GL = grau de liberdade; **, * e ns = significativo, a 1\% e 5\% de probabilidade e não significativo, respectivamente.

Tabela 5 - Relações entre densidade básica média e os valores do pilodyn e do resistógrafo.

Table 5 - Relationship among the mean basic density, the Pilodyn values, and resistograph.

\begin{tabular}{|c|c|c|c|c|c|}
\hline Modelo & & Equações & & $\mathrm{R}^{2}$ & CV (\%) \\
\hline $\begin{array}{l}\text { quadrático } \\
\text { Pilodyn }\end{array}$ & $\begin{array}{c}\hat{\beta_{0}} \\
-0,114043\end{array}$ & $\begin{array}{c}\hat{\beta_{1}} \\
0,07652\end{array}$ & $\begin{array}{c}\hat{\beta_{2}} \\
-0,002585\end{array}$ & $0,5766 * *$ & 3,18 \\
\hline Resistógrafo & 0,261448 & 0,015752 & $-0,00032$ & $0,7423 * *$ & 2,48 \\
\hline
\end{tabular}

**: Significativo, a $1 \%$ de probabilidade, $\mathrm{CV}=$ coeficiente de variação.

DB DAP x RESISTÓGRAFO

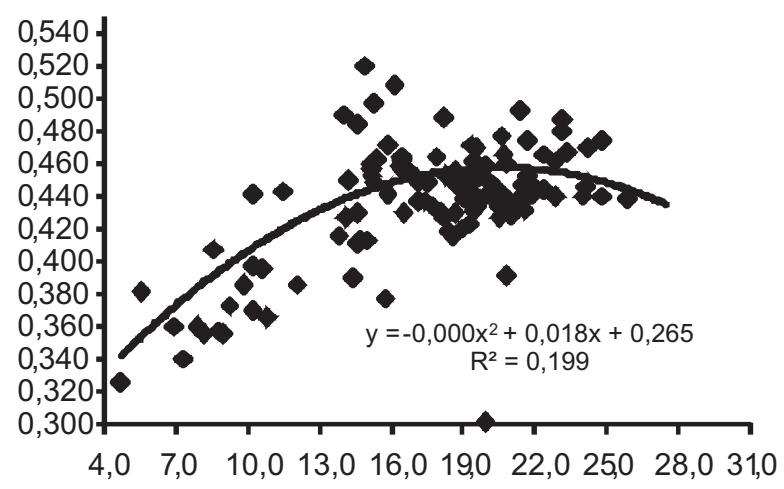

DB CAVACOS x RESISTÓGRAFO

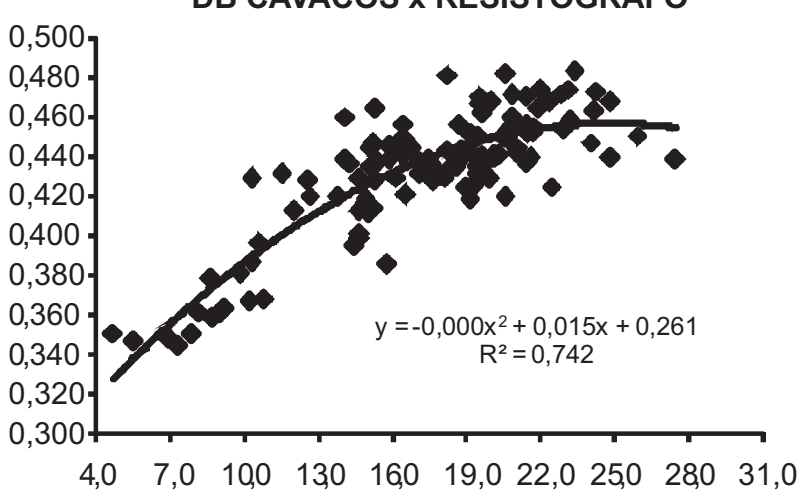

Figura 4 - Relação do resistógrafo com as densidades básicas da madeira (DbM e DbDAP).

Figure 4 - Resistograph relationship with the wood basic density (Wbd and BdDBH).

Revista Árvore, Viçosa-MG, v.35, n.2, p.349-358, 2011 
Tabela 6 - Características do modelo matemático NIRS desenvolvido para predição da densidade básica da madeira de Eucalyptus. Table 6 - NIRS mathematical model characteristics developed to predict the Eucalyptus basic density.

\begin{tabular}{cccc}
\hline Características & Fatores & $\mathrm{R}^{2}$ & RMSEP \\
\hline Densidade básica $\left(\mathrm{g} / \mathrm{cm}^{3}\right)$ & 10 & 0,84 & RMSEC \\
\hline
\end{tabular}

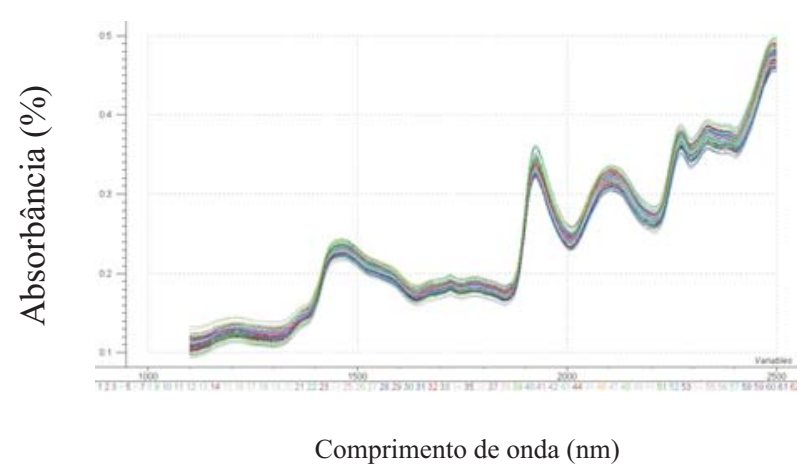

Figura 5 - Espectros NIR das 120 amostras de madeira de Eucalyptus.

Figure 5 - NIR spectra of 120 Eucalyptus samples.

parte do ajuste do modelo. Quanto mais próximo de zero, menor o erro de predição. Esse erro (RMSEP) verificado foi de somente $0,011 \mathrm{~g} / \mathrm{cm}^{3}$, demonstrando precisão perfeitamente aceitável para predizer a densidade básica da madeira de Eucalyptus com a técnica NIRS.

\section{CONCLUSÕES}

A técnica de amostragem para determinação de densidade básica das árvores utilizando discos no DAP não se apresentou adequada para estimar a densidade básica média da árvore.

O aparelho Resistograph ${ }^{\circledR}$ mostrou-se mais adequado para estimar a densidade básica da madeira, comparado ao pilodyn e ao NIRS.

O baixo erro de predição verificado com o modelo gerado pelo NIRS demonstrou ser este técnica viável para predizer a densidade básica da madeira de Eucalyptus.

\section{AGRADECIMENTOS}

À Fundação de Apoio à Pesquisa do Estado de Minas Gerais (FAPEMIG) e à CENIBRA (Celulose Nipo Brasileira S.A.), pelo apoio financeiro.

\section{6 - REFERÊNCIAS}

ASSOCIAÇÃO BRASILEIRA DE NORMAS TÉCNICADE CELULOSE E PAPEL-ABTCP. Normas. Rio de Janeiro: 2003.
CARDOSO JUNIOR, A. A. et al. Deformação residual longitudinal em diferentes espaçamentos e idades em clone de híbrido de Eucalyptus.Cerne, v.11, n.3, p.218-224. 2005.

GOMIDE, J. L. et al. Caracterização tecnológica para produção de celulose da nova geração de clones de Eucalyptus do Brasil. Revista Árvore, v.29, n.1, p.129-137. 2005.

GOMIDE, J. L.; FANTUZZI NETO, H.; LEITE, H. G. Estratégia de análise da qualidade da madeira de Eucalyptus sp para produção de celulose kraft. Revista Árvore, v.28, n.3, p.443-4507. 2004.

HEIN, P. R. G. et al. Near infrared spectroscopy for estimating wood basic density in Eucalyptus urophylla and Eucalyptus grandis. Cerne, v.15, n.2, p.133-141, 2009.

LIMA, J. T. et al. Deformações residuais longitudinais decorrentes de tensões de crescimento em Eucalyptus e suas associações com outras propriedades. Revista Árvore, v.28, n.1, p.107-116, 2004.

MILAGRES, F. R. Representatividade da densidade básica média da árvore de eucalipto determinada no DAP. In: CONGRESSO ANUAL DE CELULOSE E PAPEL, ABTCP, 42., 2008, São Paulo. Anais... São Paulo: 2008.

OLIVEIRA, L. J. R. Uso do Pilodyn para a estimativa da densidade básica e propriedades mecânicas da madeira de eucalipto. 2001. 62f. Dissertação (Mestrado em Engenharia Florestal) - Universidade Federal de Lavras, Lavras, MG.

PÁDUA, F. A. Estimativas de parâmetros genéticos das tensões de crescimento em clones de Eucalyptus. 2004. 66f. Dissertação (Mestrado em Engenharia Florestal) Universidade Federal de Lavras, Lavras, MG.

RODRIGUES, J. et al. Determination of tree to tree variation in syringyl/guaiacyl ratio of Eucalyptus globulus wood lignin analytical pyrolysis. Journal of Analytical and Applied Pyrolysis, v.48, p.121-128, 1998.

Revista Árvore, Viçosa-MG, v.35, n.2, p.349-358, 2011 
SANTOS, R. B.; GOMIDE, J. L.; SOUSA, L. C. Predição de qualidade da madeira e da polpa celulósica por técnica de espectroscopia de infravermelho próximo (NIRS). Revista Árvore, v.33, n.4, p.759-767, 2009.

SOUZA, M. A. M. et al. Deformação Residual Longitudinal (DRL) e sua relação com as características de crescimento da árvore em clones de Eucalyptus. Floresta, v.33, p.275-137, 2003.
THIERSCH, C. R. et al. O uso de modelos matemáticos na estimativa da densidade básica da madeira em plantios de clones de Eucalyptus sp. Cerne, v.12, n.3, p.264-278, 2006.

TRUGILHO, P. F. et al. Deformação residual longitudinal (DRL) e tangencial (DRT) em seis clones de Eucalyptus spp. Cerne, v.12, n.3, p.279-286, 2006. 